EPJ Web of Conferences 70, 00037 (2014)

DOI: $10.1051 /$ epjconf/ 20147000037

(C) Owned by the authors, published by EDP Sciences, 2014

\title{
Production and spectroscopy at LHCb
}

\author{
Andrea Contu ${ }^{1}$, a , on behalf of the LHCb collaboration \\ ${ }^{1}$ INFN, Sezione di Cagliari, S. P. per Sestu Km 0.700, 09042 Monserrato (CA), Italy
}

\begin{abstract}
During 2011, the LHCb experiment accumulated $\sim 1 \mathrm{fb}^{-1}$ of integrated luminosity in proton-proton collisions data at $7 \mathrm{TeV}$, collecting a sample rich in $b$ mesons and baryons. The data provide a wealth of new measurements that probes of QCD theory predictions. We present recent results in quarkonium and $b-$ and $c$ - hadron production, as well as studies of these states' properties such as masses and kinematics.
\end{abstract}

\section{Introduction}

The Large Hadron Collider Beauty experiment (LHCb) at CERN is a single-arm forward spectrometer for precision measurements of CP violation and rare decays in the $b-$ and $c$ - hadron sector [1]. The resolution for primary (secondary) vertices is $\sim 50(100) \mu \mathrm{m}$ and the momentum resolution for tracks having hits in both the VELO and the tracking stations is around 5\%. Two Ring Imaging Cherenkov (RICH) detectors provide excellent charged particle identification capabilities over a wide momentum range of $2-100 \mathrm{GeV} / c$. The tracking system is composed by a precision Vertex Locator (VELO) surrounding the interaction region, a dipole magnet and three downstream tracking stations.

Heavy quark physics studies at LHCb provide a valuable test of both perturbative and nonperturbative Quantum Chromodynamics (QCD) and of the underlying event modelling in a region complementary to other general purpose detectors. The reconstruction of $\sim 40 \%$ of the total heavy quark production is possible thanks to the unique acceptance of LHCb $(2<\eta<5)$. Moreover, the large $c \bar{c}$ and $b \bar{b}$ production cross sections in the forward region $(2<y<6)$ in $p p$ collisions, measured to be $1742 \pm 267 \mu \mathrm{b}$ [3] and $75.3 \pm 5.4_{\text {stat }} \pm 13.0_{\text {syst }} \mu \mathrm{b}$ [2] respectively, combined with the excellent detector capabilities allow for data samples of unprecedented statistical content and purity.

The results presented below are based on $1.1 \mathrm{fb}^{-1}$ of data collected by the LHCb detector during the 2010 and 2011 LHC runs.

\section{Heavy meson production and spectroscopy}

\section{$2.1 B^{+}$production cross-section}

The $B^{+}$cross-section measurement performed at $\mathrm{LHCb}$ [4] is the first in the forward region and is a powerful test of QCD@NLO. Candidate $B^{ \pm} \rightarrow J / \psi(\mu \mu) K^{ \pm}$decays are selected within a fiducial region in rapidity, $2<y<4.5$, and transverse momentum, $0<p_{T}<15 \mathrm{GeV} / c$, in which a measurement of total and differential cross-section $\left(d \sigma / d p_{T}\right)$ is performed. The mass distribution of the

\footnotetext{
ae-mail: andrea.contu@ca.infn.it
}

This is an Open Access article distributed under the terms of the Creative Commons Attribution License 2.0, which permits unrestricted use, distribution, and reproduction in any medium, provided the original work is properly cited. 
selected candidates is shown in Figure 1. The main systematic uncertainties are the tracking and the muon identification efficiencies. The differential cross-section, $d \sigma / d p_{T}$, when compared with FONLL predictions [5] (see Figure 2), shows good agreement.

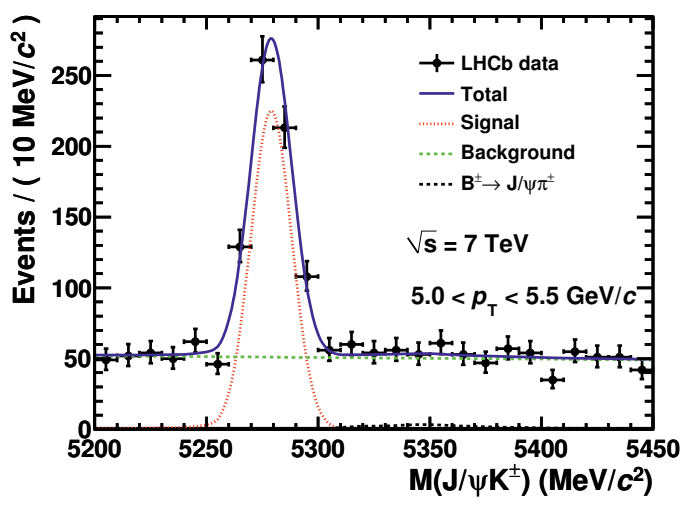

Figure 1. Invariant mass distribution of the reconstructed $B^{+}$candidates.

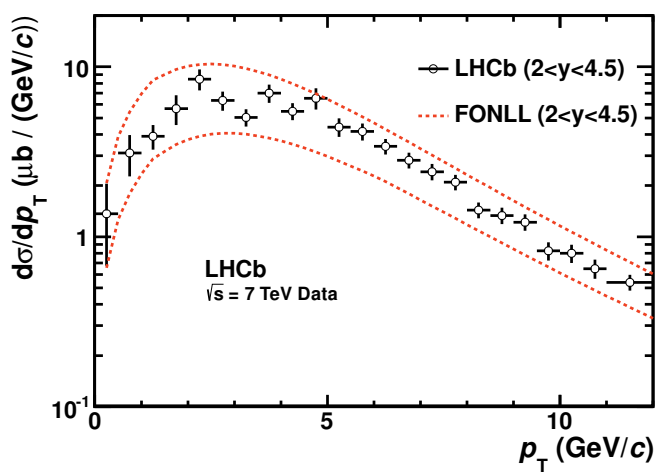

Figure 2. Cross-section as a function of the $B^{+}$transverse momentum.

\section{$2.2 B_{c}^{+}$to $B^{+}$production ratio}

Measuring the properties of the $B_{c}^{+}$mesons, such as mass, lifetime and production helps to constrain QCD calculations. The $B_{c}^{+}$meson has been first observed at CDF [6]. Since no $B_{c}^{+}$absolute branching fraction has been measured yet, the analysis performed at LHCb [7] uses $B^{ \pm} \rightarrow J / \psi K^{ \pm}$and $B_{c}^{ \pm} \rightarrow$ $J / \psi \pi^{ \pm}$candidates (see Figure 3) to perform a measurement of the production cross-section times the branching fraction of the $B_{c}^{+} \rightarrow J / \psi \pi^{+}$decay mode. We measure the ratio

$$
R_{c}^{+}=\frac{\sigma\left(B_{c}^{+}\right) \times \mathcal{B}\left(B_{c}^{+} \rightarrow J / \psi \pi^{+}\right)}{\sigma\left(B^{+}\right) \times \mathcal{B}\left(B^{+} \rightarrow J / \psi K^{+}\right)},
$$


where $\sigma\left(B_{(c)}^{+}\right)$is the production cross-section for the $B_{(c)}^{+}$meson. The measurement, performed for $B$ mesons with $p_{T}>4 \mathrm{GeV} / c$ and $2.4<\eta<4.5$, is $R_{c}^{+}=2.2 \pm 0.8 \pm 0.2$ where the first uncertainty is statistical and the second is systematic.

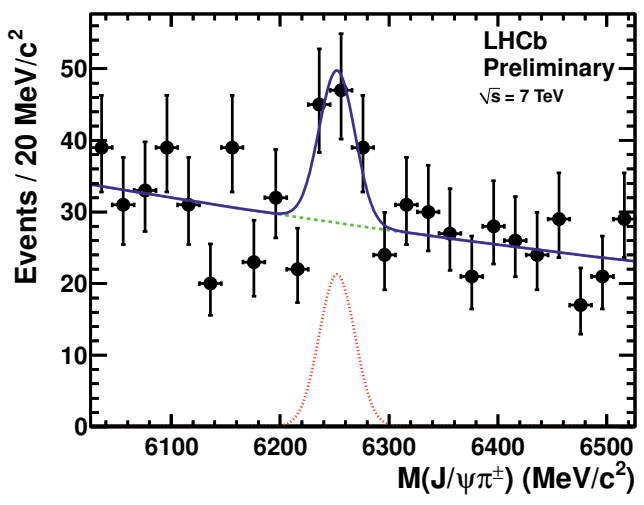

Figure 3. Invariant mass distribution for $B_{c}^{+} \rightarrow J / \psi \pi^{+}$candidates.

\subsection{First observation of $B_{c}^{+} \rightarrow J / \psi \pi^{+} \pi^{-} \pi^{+}$}

The first observation of $B_{c}^{+} \rightarrow J / \psi \pi^{+} \pi^{-} \pi^{+}$decay mode has been made by the LHCb collaboration [8]. Invariant mass distributions for selected $B_{c}^{+} \rightarrow J / \psi \pi^{+} \pi^{-} \pi^{+}$and $B_{c}^{+} \rightarrow J / \psi \pi^{+}$(used as a normalisation mode) candidates are shown in Figure 4 . The analysis of the resonant structures show that the domi-

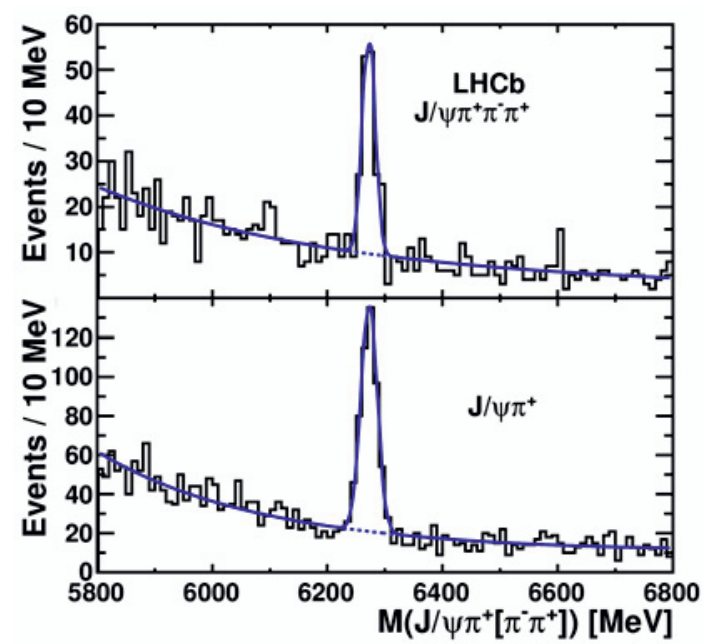

Figure 4. Invariant mass distribution of $B_{c}^{+} \rightarrow J / \psi \pi^{+} \pi^{-} \pi^{+}$(top) and $B_{c}^{+} \rightarrow J / \psi \pi^{+}$(bottom) candidates.

nant contribution is from $B_{c}^{+} \rightarrow J / \psi a_{1}^{+}$, where $a_{1}^{+} \rightarrow \rho^{0} \pi^{+}$and is in good agreement with theoretical 
predictions from the BLL model described in Ref.[9]. The relative branching fraction with respect to the single pion mode has been measured to be

$$
\frac{\mathcal{B}\left(B_{c}^{+} \rightarrow J / \psi \pi^{+} \pi^{-} \pi^{+}\right)}{\mathcal{B}\left(B_{c}^{+} \rightarrow J / \psi \pi^{+}\right)}=2.41 \pm 0.30_{\text {stat }} \pm 0.33_{\text {syst }} .
$$

\subsection{Orbitally exited $B^{* *}$ mesons observation}

Orbitally excited $B_{(s)}$ mesons containing a light quark, hereafter collectively referred to as $B^{* *}$ mesons, are predicted by the Heavy Quark Effective Theory (HQET) [10] in the limit of infinite $b$-quark mass. Several of these states have been already observed at the Tevatron by the CDF [11] and D0 [12, 13] experiments. A search for $B^{* *} \rightarrow B h$ and $B^{* *} \rightarrow B^{*} \gamma$ has been performed using $336 \mathrm{pb}^{-1}$ of data collected in 2011. Soft photons from the $B^{* 0}$ decay are not reconstructed, therefore, signal peaks are expected to appear in the relative to threshold invariant mass

$$
Q=M(B h)-M(B)-M(h) .
$$

Distributions for $Q$, where signal resonance distributions are fitted using relativistic Breit-Wigner shapes, are shown in Figure 5-7. The main systematic uncertainties affecting the measured $Q$ values

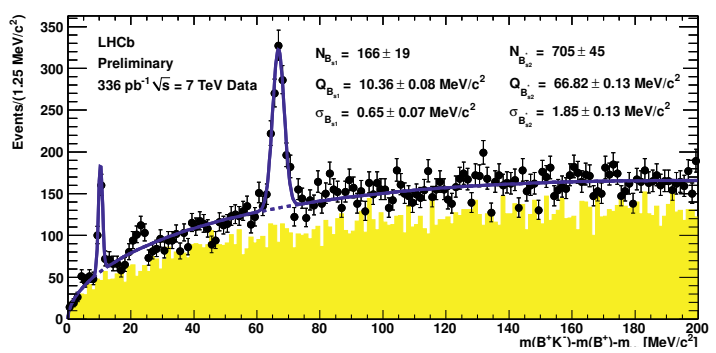

Figure 5. $Q\left(B^{+} K^{-}\right)$distribution. LHCb data (black markers) and the total fit are superimposed.

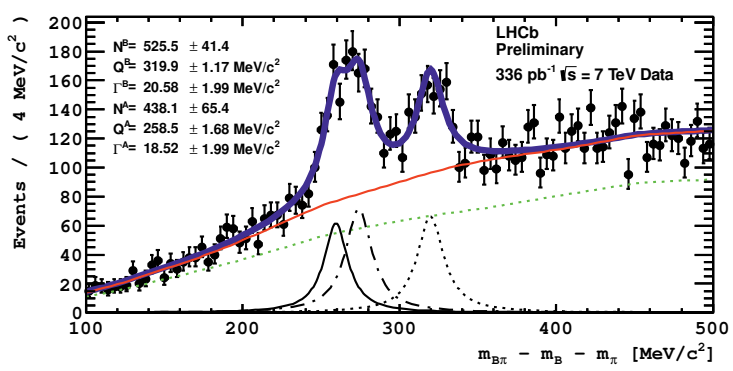

Figure 6. $Q\left(B^{+} \pi^{-}\right)$distribution. LHCb data (black markers) and the total fit are superimposed. Individual components contributing to the total fit are combinatorial background and associated production (red, green for combinatorial background only), $B_{1}^{0} \rightarrow B^{*+} \pi^{-}$(solid black), $B_{2}^{* 0} \rightarrow B^{*+} \pi^{-}$(dot-dashed black) and $B_{2}^{* 0} \rightarrow B^{+} \pi^{-}$. 


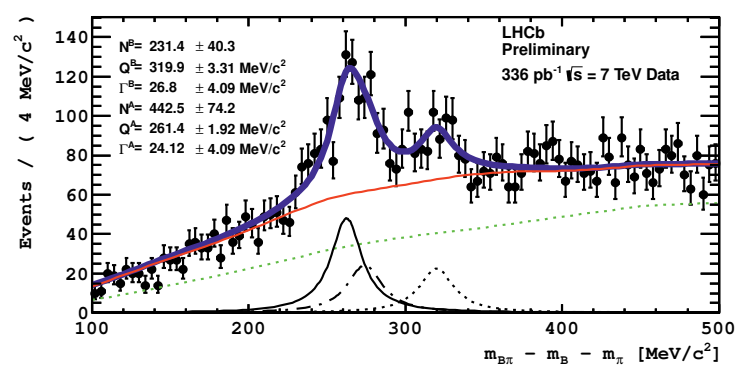

Figure 7. $Q\left(B^{0} \pi^{+}\right)$distribution. LHCb data (black markers) and the total fit are superimposed. Individual components contributing to the total fit are combinatorial background and associated production (red, green for combinatorial background only), $B_{1}^{+} \rightarrow B^{* 0} \pi^{+}$(solid black), $B_{2}^{*+} \rightarrow B^{* 0} \pi^{+}$(dot-dashed black) and $B_{2}^{*+} \rightarrow B^{0} \pi^{+}$.

arise from the variation of selection requirements and the uncertainty on the $B^{0}$ mass. The measured $Q$ values can be translated into masses to give the following results

$$
\begin{aligned}
M_{B_{s 1}^{0}} & =\left(5828.99 \pm 0.8_{\text {stat }} \pm 0.13_{\text {syst }} \pm 0.45_{\text {syst }}^{\text {Bmass }}\right) \mathrm{MeV} / c^{2} \\
M_{B_{s 2}^{* 0}} & =\left(5839.67 \pm 0.13_{\text {stat }} \pm 0.17_{\text {syst }} \pm 0.29_{\text {syst }}^{\text {Bmass }}\right) \mathrm{MeV} / c^{2} \\
M_{B_{1}^{0}} & =\left(5724.1 \pm 1.7_{\text {stat }} \pm 2.0_{\text {syst }} \pm 0.5_{\text {syst }}^{\text {Bmass }}\right) \mathrm{MeV} / c^{2} \\
M_{B_{1}^{+}} & =\left(5726.3 \pm 1.9_{\text {stat }} \pm 3.0_{\text {syst }} \pm 0.5_{\text {syst }}^{\text {Bmass }}\right) \mathrm{MeV} / c^{2} \\
M_{B_{2}^{* 0}} & =\left(5738.6 \pm 1.2_{\text {stat }} \pm 1.2_{\text {syst }} \pm 0.3_{\text {syst }}^{\text {Bmass }}\right) \mathrm{MeV} / c^{2} \\
M_{B_{2}^{*+}} & =\left(5739.0 \pm 3.3_{\text {stat }} \pm 1.6_{\text {syst }} \pm 0.3_{\text {syst }}^{\text {Bass }}\right) \mathrm{MeV} / c^{2}
\end{aligned}
$$

The $B_{2}^{*+}$ and $B_{1}^{+}$states are observed for the first time with a signal significance of $4.0 \sigma$ and $9.9 \sigma$ respectively. It is also important to notice that measured masses are in agreement with HQET prediction and that isospin partners have compatible masses.

\section{Heavy baryon spectroscopy}

While $b$-mesons are relatively well known objects, very little is known about $b$-baryons. There are seven ground-state $\left(J^{P}=1 / 2^{+}\right)$baryons involving a $b$ quark and two light quarks $(u, d, s)$. These states can be ordered in a triangular scheme as shown in Figure 8. In the following section we will investigate properties of these baryons and their orbitally excited states.

\subsection{Beauty baryons decaying to $D^{0} p K^{-}$and $D^{0} p \pi^{-}$}

Decays of $\Lambda_{b}^{0}$ and $\Xi_{b}^{0}$ into the $D^{0} p K^{-}$and $D^{0} p \pi^{-}$final states are potentially useful to improve the precision on the CKM angle $\gamma[18,19]$. We selected $\Lambda_{b}^{0}$ and $\Xi_{b}^{0}$ candidates undergoing the above decay chain using decay topology and particle identification in a data sample of $330 \mathrm{fb}^{-1}$ [17]. We performed several measurements involving these decays. In particular, we measured the ratio

$$
\begin{aligned}
\frac{\mathcal{B}\left(\Lambda_{b}^{0} \rightarrow D^{0} p \pi^{-}\right) \times \mathcal{B}\left(D^{0} \rightarrow K^{-} \pi^{+}\right)}{\mathcal{B}\left(\Lambda_{b}^{0} \rightarrow \Lambda_{c}^{+} \pi^{-}\right) \times \mathcal{B}\left(\Lambda_{c}^{+} \rightarrow p K^{-} \pi^{+}\right)}= \\
\quad=0.119 \pm 0.006_{\text {stat }} \pm 0.013_{\text {syst }}
\end{aligned}
$$




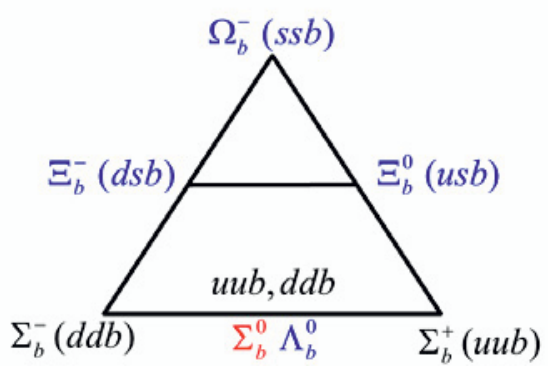

Figure 8. Ground-state $b$-baryons in a triangular arrangement. The isospin and the strangeness quantum numbers run on the horizontal and vertical axis respectively.

and report the first observation of the decay $\Lambda_{b}^{0} \rightarrow D^{0} p K^{-}$, for which a mass peak is clearly visible in Figure 9. The $\Xi_{b}^{0}$ has already been observed at CDF [16] and the mass measured to be $5787.8 \pm 5.5_{\text {stat }} \pm$

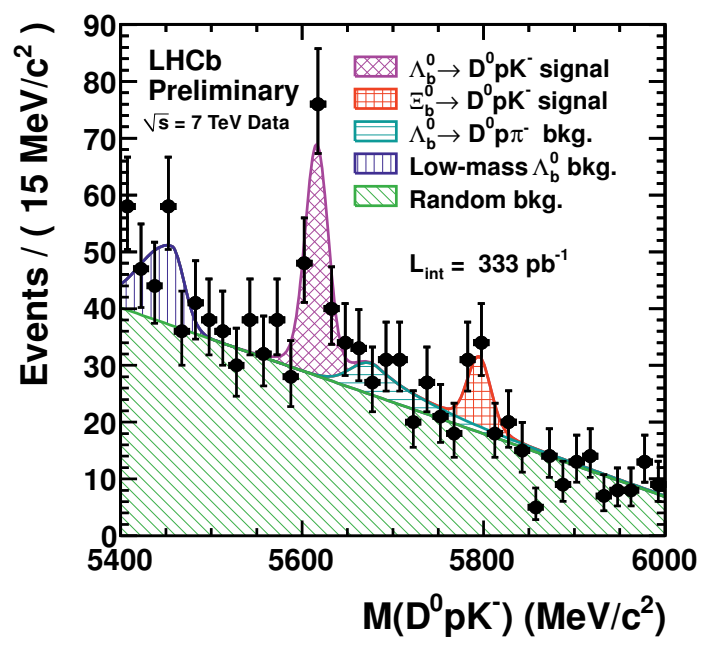

Figure 9. Invariant mass distribution of the selected $D^{0} p K^{-}$combinations.

$0.5_{\text {syst }}$. The $\Xi_{b}^{0}$ mass measurement performed at LHCb of $5802.0 \pm 5.5_{\text {stat }} \pm 1.7_{\text {syst }}$ is compatible.

\subsection{Measurement of $\Xi_{b}^{-}$and $\Omega_{b}^{-}$masses}

Previous measurements of the $\Xi_{b}^{-}$and $\Omega_{b}^{-}$masses were performed at the Tevatron by the CDF [22] and D0 [23] experiments. The same measurement has been repeated at LHCb [20] using reconstructed $\Xi_{b}^{-}$and $\Omega_{b}^{-}$candidates decaying into $J / \psi \Xi^{-}\left(\Lambda^{0} K^{-}\right)$and $J / \psi \Omega^{-}\left(\Lambda^{0} \pi^{-}\right)$respectively, with $J / \psi \rightarrow \mu^{+} \mu^{-}$ and $\Lambda^{0} \rightarrow p \pi^{-}$. Having three displaced vertices, the decay topology is rich, as outlined in Figure 10. The invariant mass distributions for the selected candidates are shown in Figure 11-12. The signal is described by a Gaussian functions in which the $\Xi_{b}^{-}$width is extracted from simulation while the $\Omega_{b}^{-}$ width is estimated by scaling the $\Xi_{b}^{-}$width to the ratio of the $\Omega_{b}^{-}$to $\Xi_{b}^{-}$masses. The results for the 


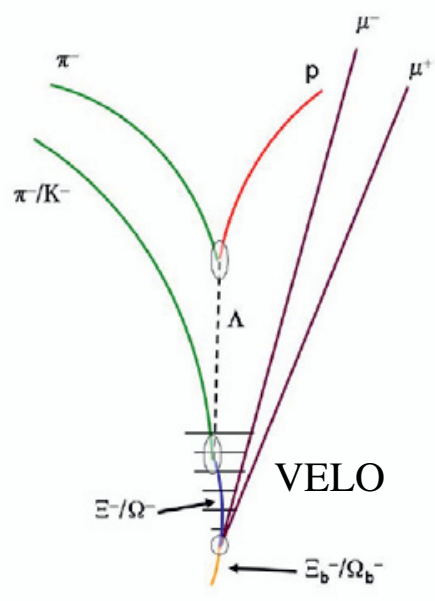

Figure 10. Scheme of the $\Xi_{b}^{-}\left(\Omega_{b}^{-}\right)$decay. In this particular example two decay vertices are located inside the VELO while the $\Lambda$ vertex is located outside due to its long lifetime.

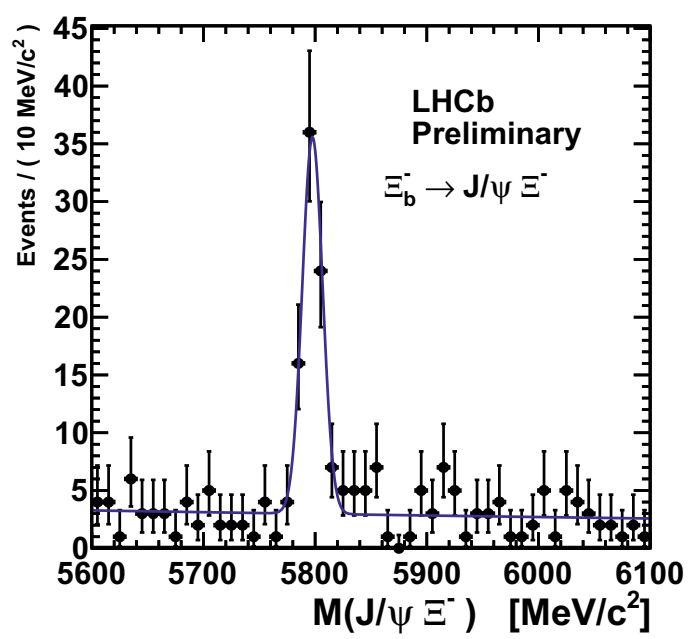

Figure 11. $\Xi_{b}^{-}$candidates invariant mass distribution.

masses, the best to date, are

$$
\begin{aligned}
& M\left(\Xi_{b}^{-}\right)=\left(5796.5 \pm 1.2_{\text {stat }} \pm 1.2_{\text {syst }}\right) \mathrm{MeV} / c^{2}, \\
& M\left(\Omega_{b}^{-}\right)=\left(6050.3 \pm 4.5_{\text {stat }} \pm 2.2_{\text {syst }}\right) \mathrm{MeV} / c^{2},
\end{aligned}
$$

where the systematic uncertainties are dominated by the calibration of the momentum scale [21]. While LHCb's $\Xi_{b}^{-}$mass measurement is consistent with both CDF and DO results, we favour CDF result for the $\Omega_{b}^{-}$mass $\left(6054.4 \pm 6.9 \mathrm{MeV} / c^{2}\right)$ with respect to the one from D0 $\left(6165 \pm 16 \mathrm{MeV} / c^{2}\right)$. 


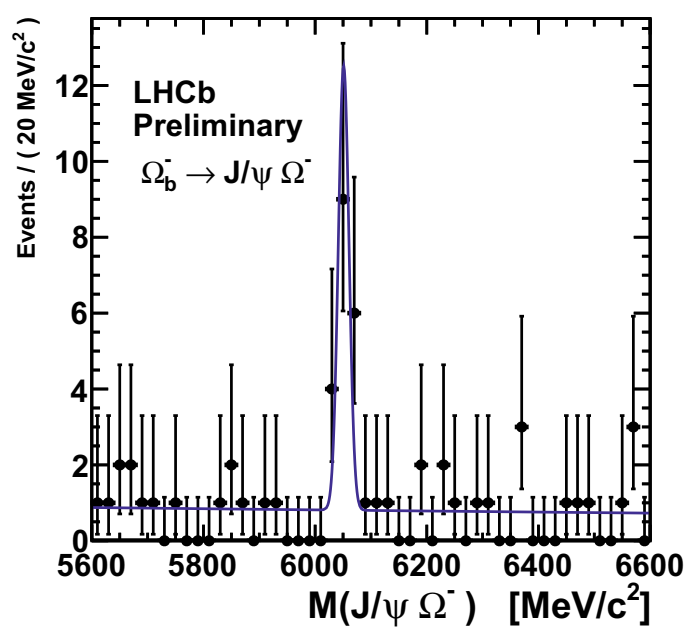

Figure 12. $\Omega_{b}^{-}$candidates invariant mass distribution.

\subsection{First observation of $\Lambda_{b}^{0 *}$}

Orbitally excited $\Lambda_{b}^{0}$ states having $J^{P}=1 / 2^{-}$and $J^{P}=3 / 2^{-}$are predicted in several scenarios [15]. These states are expected to decay into $\Lambda_{0}$ via radiative or dipion decay. We performed a search for new states in the $\Lambda_{b}^{0} \pi^{+} \pi^{-}$invariant mass using $1 \mathrm{fb}^{-1}$ of data from the 2011 run [14]. The invariant mass distributions for the $\Lambda_{b}^{0}$ and $\Lambda_{b}^{0 *}$ candidates are shown in Figure 13 and Figure 14 respectively. The two mass peaks in the $\Lambda_{b} \pi^{+} \pi^{-}$invariant mass have been described using a Gaussian distribution. The background model is extracted from the invariant mass distribution of wrong-sign $\Lambda_{b}^{0} \pi^{+} \pi^{+}$combinations. The mass values have been fitted to be

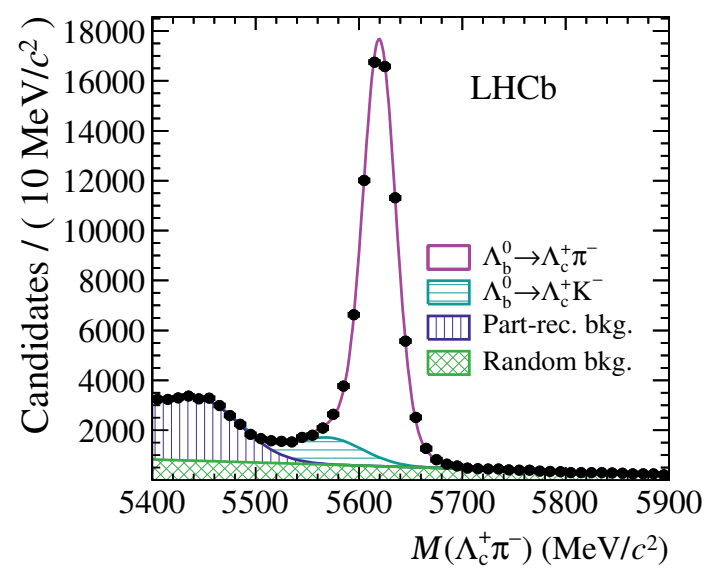

Figure 13. Invariant mass distribution for $\Lambda_{b}^{0} \rightarrow \Lambda_{c}^{+} \pi^{-}$candidates. 


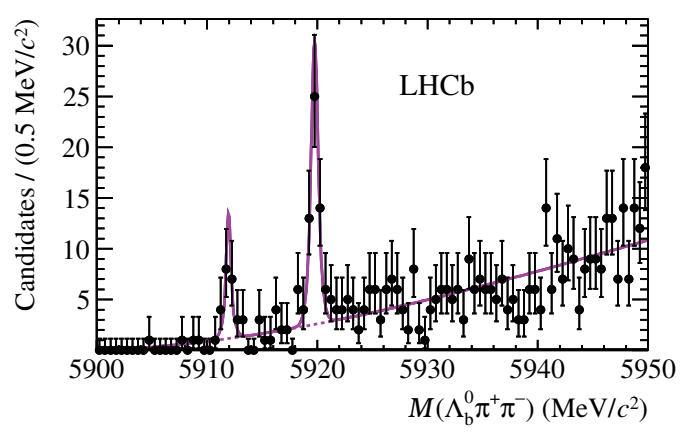

Figure 14. Invariant mass distribution for $\Lambda_{b}^{0 *} \rightarrow \Lambda_{b}^{0} \pi^{+} \pi^{-}$candidates.

$$
\begin{aligned}
& 5911.95 \pm 0.12_{\text {stat }} \pm 0.03_{\text {syst }} \pm 0.66_{\Lambda_{b}^{0} \text { mass }}, \\
& 5919.76 \pm 0.07_{\text {stat }} \pm 0.02_{\text {syst }} \pm 0.66_{\Lambda_{b}^{0} \text { mass }} .
\end{aligned}
$$

Therefore, these states can be interpreted as the states $\Lambda_{b}^{* 0}(5912)$ and $\Lambda_{b}^{* 0}(5920)$.

\section{4 c-hadrons}

\subsection{Double charm production}

Studies of double charmonium and charmonium with associated open charm production can be a useful probe of the quarkonium production mechanism. In addition, contributions from other mechanisms, such as Double Parton Scattering (DPS) may be investigated [28, 29]. A search for the production of an open charm hadron $\left(D^{0}, D^{+}, D_{s}^{+}, \Lambda_{c}^{+}\right)$, hereafter generically referred to as $C$, in association with a $J / \psi$ meson or another open charm hadron has been performed at $\mathrm{LHCb}$ using $355 \mathrm{pb}^{-1}$ of data collected in 2011 [24]. Examples two-dimensional mass distribution are shown in Figure 15 for the selected candidates.

Absolute cross-sections and cross-section ratios have been measured and compared with theoretical expectations, as shown in Figure 16-17. In addition, the properties of $J / \psi C, C C$, and $C \bar{C}$ events, such as transverse momentum spectra, have been studied (see an example plot in Figure 18).

\section{$4.2 \chi_{c}$ production}

Radiative decays of the P-wave charmonia $\chi_{c J}(J=1,2,3)$ give a substantial feed-down contribution to $J / \psi$ production and have a major impact on the $J / \psi$ polarisation. We present a measurement of the production cross-section ratio of $\chi_{c 2}$ to $\chi_{c 1}[30,31]$, which is sensitive to colour-singlet and colouroctet production mechanisms. The identification of the $\chi_{c}$ is obtained via the reconstruction of its radiative decay into $J / \psi \gamma$. The analysis has been performed separately for converted and unconverted photons. Converted photons interacted with the detector material late in the tracking system and converted into an $e^{+} e^{-}$pair. Both converted and unconverted categories are measured using calorimeter information alone.

Mass distribution are shown separately when using converted and unconverted photons in Figure 19. The results are shown in Figure 20 and compared to CDF [32] results (at $\sqrt{s}=1.96 \mathrm{TeV}$ in $p \bar{p}$ collisions and in the $J / \psi$ pseudo-rapidity range $\left|\eta^{J / \psi}\right|<1.0$ ), NLO NRQCD [33] and ChiGen 

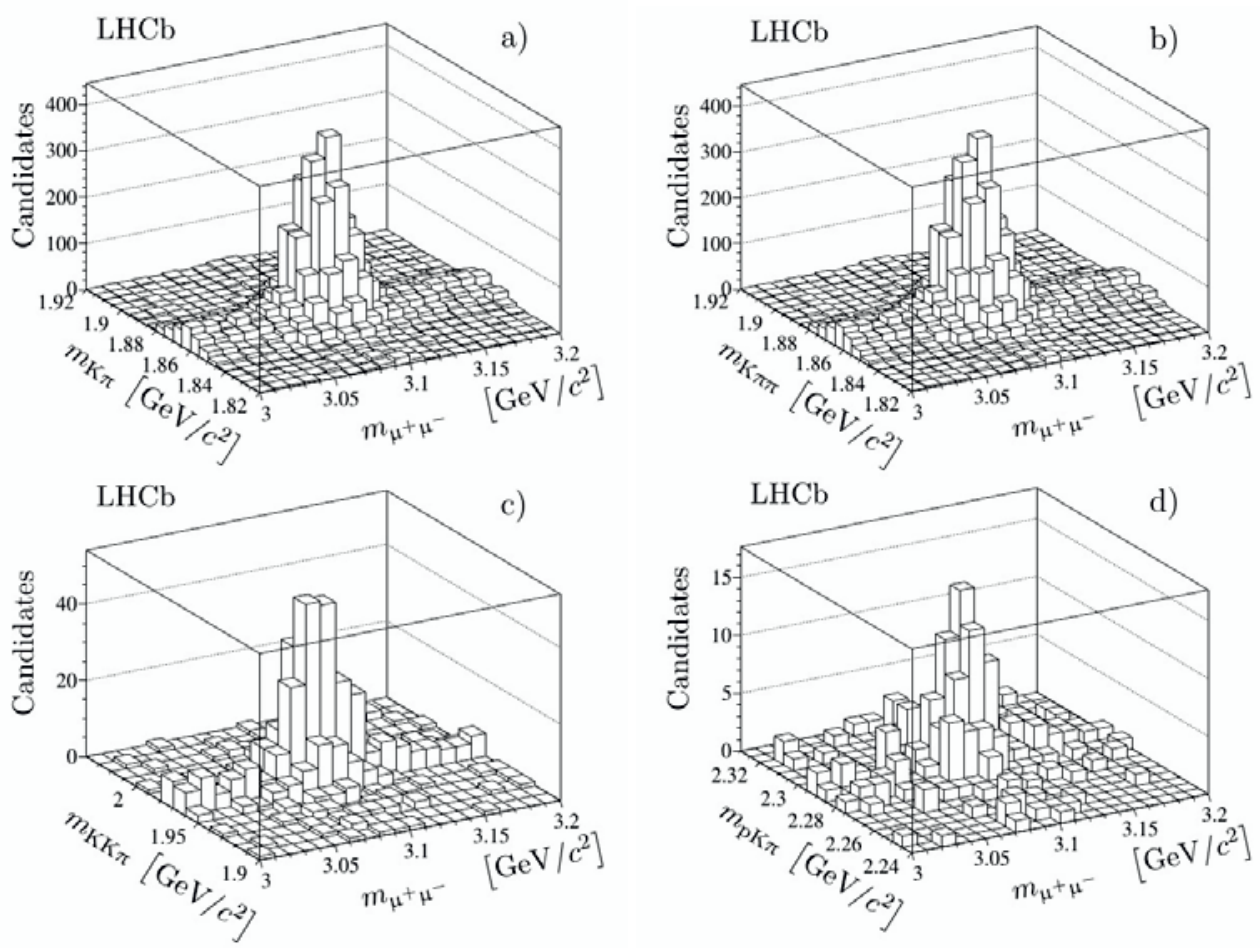

Figure 15. Invariant mass distributions for a) $J / \psi D^{0}$, b) $J / \psi D^{+}$, c) $J / \psi D_{s}^{+}$and d) $J / \psi \Lambda_{c}^{+}$candidates.

Monte Carlo generator [34] predictions. It can be noted that the ChiGen generator describes the shape of the distribution reasonably well, although the data lie consistently above the model prediction. The results are in agreement with NLO NRQCD predictions only for $p_{T}^{J / \psi}>8 \mathrm{GeV} / c$.

\section{Conclusions}

We presented selected measurement in the heavy flavour sector performed by the LHCb collaboration. The outstanding capabilities and statistical power of the LHCb detector produced competitive results on heavy baryon production and spectroscopy. New results are expected from the analysis of the full 2011 sample and the 2012 data. 
ICFP 2012

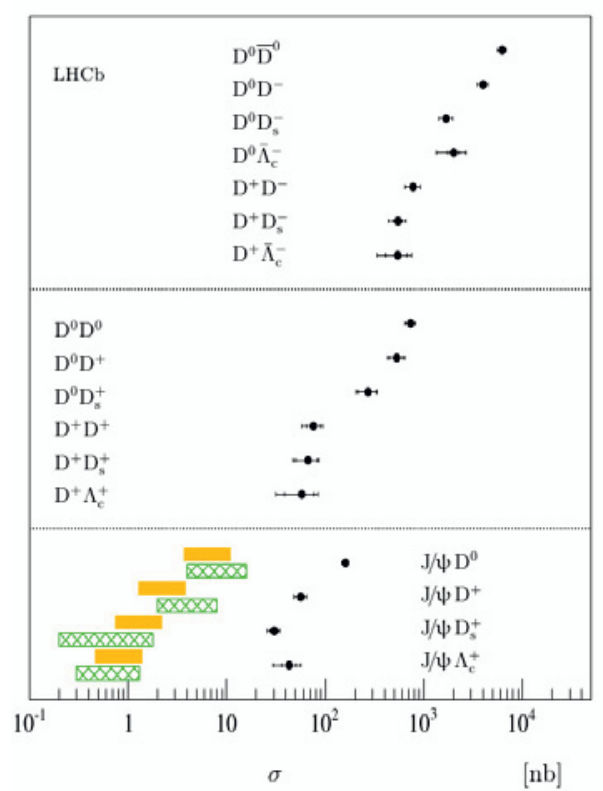

Figure 16. Measured cross-sections $\sigma_{J / \psi} C, \sigma_{C C}$ and $\sigma_{C C}$ (points with error bars) compared, in $J / \psi C$ channels, to the calculations in $[25,26]$ (hatched areas) and [27] (shaded areas). The inner error bars indicate the statistical uncertainty whilst the outer error bars indicate the sum of the statistical and systematic uncertainties in quadrature. Charge-conjugate modes are included. 


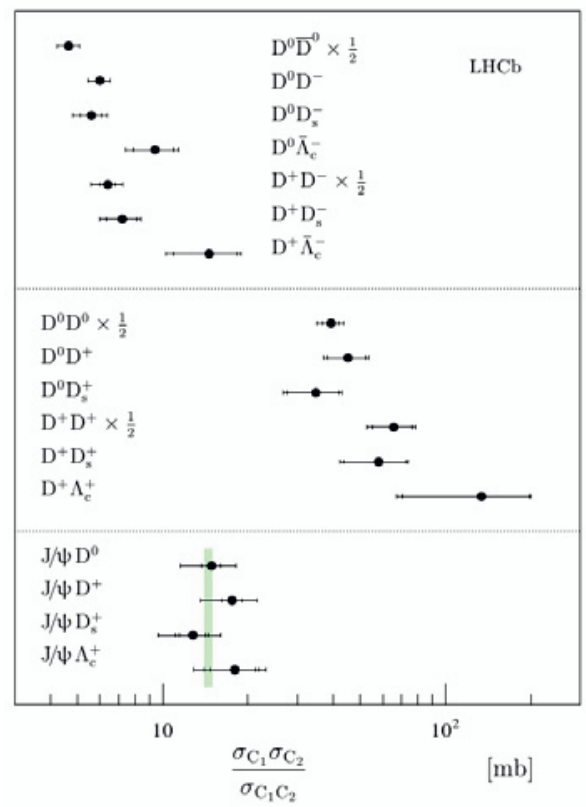

Figure 17. Measured ratios $\sigma_{C_{1}} \sigma_{C_{2}} / \sigma_{C_{1} C_{2}}$ (points with error bars) in comparison with the expectations from DPS using the cross-section measured at Tevatron for multi-jet events (light green shaded area). For the $D^{0} D^{0}$, $D^{0} \bar{D}^{0}, D^{+} D^{+}$and $D^{+} D^{-}$cases the ratios are rescaled with the symmetry factor of one half. The inner error bars indicate the statistical uncertainty whilst the outer error bars indicate the sum of the statistical and systematic uncertainties in quadrature. For the $J / \psi C$ case the outermost error bars correspond to the total uncertainties including the uncertainties due to the unknown polarization of the prompt $J / \psi$ mesons.
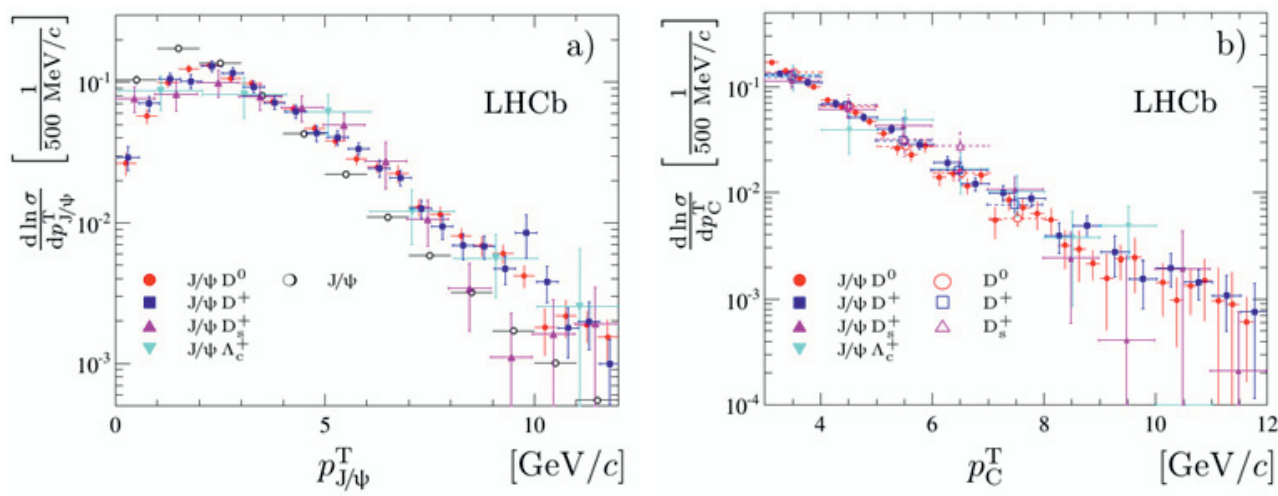

Figure 18. a) Transverse momentum spectra of $J / \psi$ for $J / \psi C$ and prompt $J / \psi$ events. b) Transverse momentum spectra for open charm hadrons for $J / \psi C$ and prompt $D^{0}, D^{+}$and $D_{s}^{+}$events. 

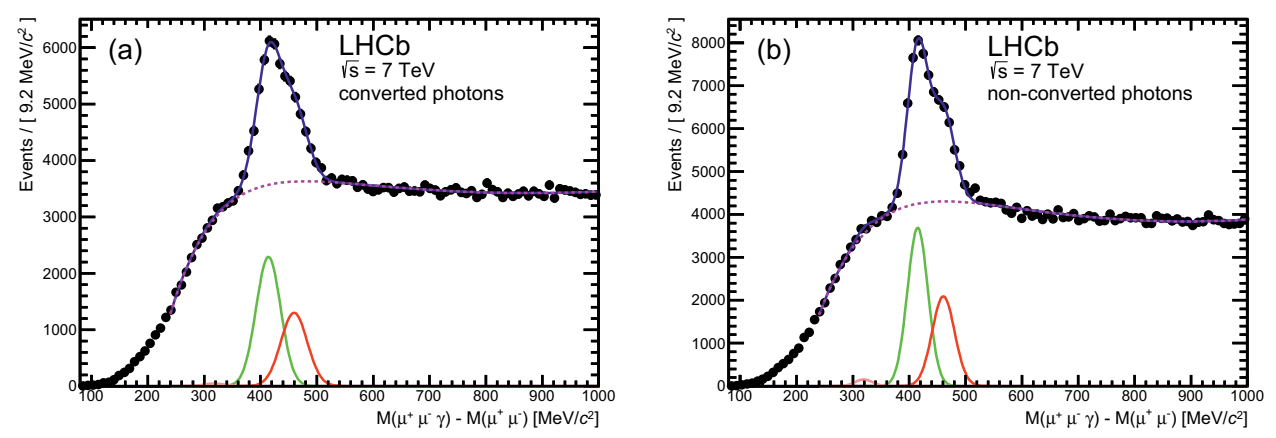

Figure 19. Distribution of the mass difference $\Delta M=M\left(\mu^{+} \mu^{-} \gamma\right)-M\left(\mu^{+} \mu^{-}\right)$for selected candidates with $3<$ $p_{T}^{J / \psi}<15 \mathrm{GeV} / c$ for (a) converted and (b) non-converted photons. The lower solid curves correspond to the $\chi_{c 0}$ ,$\chi_{c 1}$ and $\chi_{c 2}$ peaks from left to right, respectively. The background distribution is shown as a dashed curve. The upper solid curve corresponds to the overall fit function. 

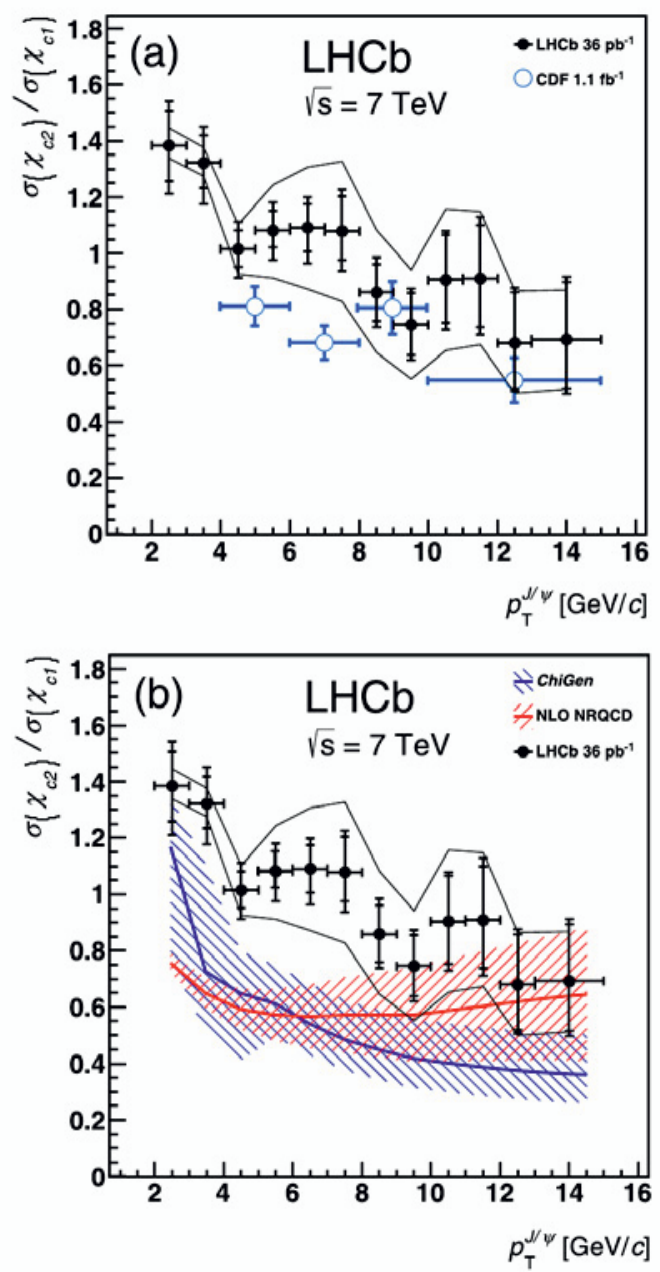

Figure 20. Ratio $\sigma\left(\chi_{2 c}\right) / \sigma\left(\chi_{c 1}\right)$ in bins of $2<p_{T}^{J / \psi}<15 \mathrm{GeV} / c$. The LHCb results, in the rapidity range $2.0<y^{J / \psi}<4.5$ and assuming the production of unpolarised $\chi_{c}$ mesons, are shown with solid black circles and the internal error bars correspond to the statistical error; the external error bars include the contribution from the systematic uncertainties (apart from the polarisation). The lines surrounding the data points show the maximum effect of the unknown $\chi_{c}$ polarisations on the result. The upper and lower limits correspond to the spin states as described in the text. The CDF data points are superimposed in (a) with open blue circles [32]. The two hatched bands in (b) correspond to the ChiGen Monte Carlo generator [34] and NLO NRQCD [33] predictions. 


\section{References}

[1] The LHCb Collaboration, JINST 3, (2008) S08005

[2] The LHCb Collaboration, Phys. Lett. B694, (2010) 209

[3] The LHCb Collaboration, LHCb-CONF-2010-013

[4] The LHCb Collaboration, JHEP 04, (2012) 093

[5] M. Cacciari et al, JHEP 05, (2008) 007

[6] The CDF Collaboration, Phys. Rev. Lett. 81, (1998) 2432

[7] The LHCb Collaboration, LHCb-CONF-2011-017

[8] The LHCb Collaboration, LHCb-PAPER-2011-044, arXiv:1204.0079

[9] A. Rakitin et al, Phys. Rev. D81, (2010) 014015

[10] M. Di Pierro and E. Eichten, Phys. Rev. D64, (2001) 114004

[11] The CDF Collaboration, Phys. Rev. Lett. 102, (2009) 102003

[12] The D0 Collaboration, Phys. Rev. Lett. 99, (2007) 172001

[13] The D0 Collaboration, Phys. Rev. Lett. 100, (2008) 082001/082002

[14] The LHCb Collaboration, arxiv:1205.3452

[15] S. Capstick and N. Isgur, Phys. Rev. D34, (1986) 2809. Z. Aziza Baccouche, C. -K. Chow, T. D. Cohen and B. A. Gelman, Nucl. Phys. A696, (2001) 638. H. Garcilazo, J. Vijande and A. Valcarce, J. Phys. G G 34, (2007) 961. D. Ebert, R. N. Faustov and V. O. Galkin, Phys. Lett. B659, (2008) 612. W. Roberts and M. Pervin, Int. J. Mod. Phys. A23, (2008) 2817. M. Karliner, B. Keren-Zur, H. J. Lipkin and J. L. Rosner, Annals Phys. 324, (2009) 2. S. Narison and R. Albuquerque, Phys. Lett. B694, (2010) 217. R. M. Albuquerque, S. Narison and M. Nielsen, Phys. Lett. B684 (2010) 236.

[16] The CDF Collaboration, Phys. Rev. Lett. 107, (2011) 102001

[17] The LHCb Collaboration, LHCb-CONF-2011-036

[18] N. Cabibbo, Phys. Rev. Lett. 10, (1963) 531

[19] M. Kobayashi and T. Maskawa, Prog. Theor. Phys. 49, (1973) 652

[20] The LHCb Collaboration, LHCb-CONF-2011-060

[21] The LHCb Collaboration, LHCb-CONF-2011-027

[22] The CDF Collaboration, Phys. Rev. D80, (2009) 072003

[23] The CDF Collaboration, Phys. Rev. Lett. 101, (20098) 232002

[24] The LHCb Collaboration, JHEP 06, (2012) 141

[25] A. Berezhnoy, V. Kiselev, A. Likhoded and A. Onishchenko, Phys. Rev. D57, (1998) 4385

[26] S. Baranov, Phys. Rev. D73, (2006) 074021

[27] J. Lansberg, Eur. Phys. J. C61, (2009) 693

[28] A. Novoselov, arXiv:1106.2184

[29] M. Luszczak, R. Maciula and A. Szczurek,arXiv:1111.3255

[30] The LHCb Collaboration, Phys. Lett. B714, (2012) 215-223

[31] The LHCb Collaboration, LHCb-CONF-2011-062

[32] The CDF Collaboration, Phys. Rev. Lett. 98, (2007) 232001

[33] Y.-Q. Ma, K. Wang, K.-T. Chao, Phys. Rev. D83, (2011) 111503

[34] L.A. Harland-Lang, W.J. Stirling, http://projects.hepforge.org/superchic/chigen.html 